\title{
Alveolar macrophage dysfunction in malignant lung tumours
}

\author{
E LEMARIE, P CARRE, MF LEGRAND, M LAVANDIER, E BOISSINOT, M RENOUX, \\ G RENOUX \\ From the Service de Pneumologie and the Laboratoire d'Immunologie, Centre Hospitalier Universitaire, \\ Tours, France
}

\begin{abstract}
Alveolar macrophage chemotaxis was measured in 129 individuals -13 normal volunteers, 15 tumour free patients with recent bronchopulmonary infections, 10 patients with chronic bronchitis, 29 patients with endothoracic sarcoidosis, 48 patients with primary bronchial carcinoma and 14 patients with pulmonary metastases from various origins. Chemotaxis was tested in the presence of either zymosan activated autologous serum, $N$ formyl-methionine-leucyl-phenylalanine (F-Met-Leu-Phe), or zymosan activated human AB serum. Alveolar macrophage chemotaxis was significantly less in patients with bronchial carcinoma than in healthy volunteers $(p<0.01)$. Chemotaxis was significantly more depressed in samples obtained from the neighbourhood of the tumour than in samples from the opposite lung. Defective chemotaxis was also found in patients with sarcoidosis. In contrast, the presence of lung metastases did not affect chemotaxis. A recent bronchopulmonary infection was associated with significantly increased $(p<0.02)$ chemotaxis in tumour free patients but not in patients with a primary lung tumour. The findings suggest that an intrinsic functional defect of alveolar macrophages might favour the development of bronchogenic carcinoma.
\end{abstract}

The critically important role of alveolar macrophages in the defence of the host against inhaled particles, including microorganisms and environmental toxins, has been well established.' Yet the function of alveolar macrophages in protecting against tumour growth is still poorly understood. Several reports recently reviewed by Kaltreider ${ }^{2}$ give evidence for a role of alveolar macrophages within immunologically mediated host defence mechanisms, leading to either activation or suppression of mitogen induced lymphocyte proliferation, depending on the experimental conditions. It is well established also that macrophages or monocytes from sources other than the lung play an important part in antitumour immunity, ${ }^{34}$ their chemotaxis being frequently impaired in patients with various types of neoplasia. ${ }^{5-9}$ Chemotaxis along an attractant gradient is an important function of macrophages which allows them to reach the site of an infection or tumour.

Address for reprint requests: Dr E Lemarie, Service de Pneumologie, CHU Bretonneau, 37044 Tours Cedex, France.

Accepted 23 January 1984
We therefore thought it of interest to study the in vitro chemotactic activity of alveolar macrophages from patients with bronchial carcinoma or pulmonary metastases and compare it with that of alveolar macrophages from patients with other lung diseases and from healthy volunteers. We report here an intrinsic chemotactic defect in alveolar macrophages from patients with primary lung cancer and sarcoidosis, which was not found in cells sampled from patients with the other conditions we tested.

\section{Methods}

\section{PATIENTS}

Informed consent was obtained from the 129 individuals who took part in this study.

Group 1 consisted of 13 healthy volunteers: eight men and five women (mean age 30 years) with no known past history of lung disease, of whom eight were smokers and five non-smokers. Group 2 comprised 15 men (mean age 52, range 29-72 years). Twelve were smokers and three were non-smokers. All underwent bronchoscopic assessment after treatment as part of the investigation of longstand- 
ing or recent bronchopulmonary infection. Group 3 was made up of 10 men aged 37-69 years (mean 51) with non-infective chronic bronchitis. Six were smokers, four were non-smokers. Group 4 consisted of 29 untreated patients with endothoracic sarcoidosis-17 men and 12 women aged 19-56 years (mean 34). Five were smokers and 24 were non-smokers. Group 5 consisted of 48 patients-44 men and four women aged 40-73 years (mean 59) with primary bronchogenic carcinoma. Forty three were smokers and five were non-smokers. Thirty individuals had intrathoracic disease only. Eighteen patients had distant metastases. The histological types were: 28 squamous cell carcinomas, six small cell cancers, six adenocarcinomas, and eight undifferentiated tumours. Fourteen of the 48 patients had a concomitant infection or severe local inflammation. According to the diagnostic criteria for inflammation and infection, there had to be an inflamed bronchial mucosa at bronchoscopy associated with either leucocytosis (polymorphonuclear leucocytes over $\left.10.0 \times 10^{\%} / 1\right)$, fever higher than $38^{\circ} \mathrm{C}$, or other systemic features of inflammation (fibrinaemia $>4.5 \mathrm{~g} / \mathrm{l}$ ). Group 6 comprised 14 patients: nine men and five women aged 35-70 years (mean 55) with pulmonary metastases from primary tumours originating in the breast (5), head and neck (4), testis (1), or colon (1); in three the site of the primary tumour was unknown. Six were smokers and eight non-smokers.

\section{ALVEOLAR LAVAGE}

Segmental lavage through a fibreoptic bronchoscope was performed under local anaesthesia as previously described. ${ }^{10}$ In brief, healthy volunteers of group 1 and control patients of groups 2, 3, and 4 underwent lavage in the lingular territory. In the patients with primary bronchogenic carcinoma (group 5) alveolar lavage was performed before specific treatment at the tumour site in 23 patients. A bordering territory was lavaged in the other 25 patients because bronchial obstruction by the tumour had rendered injection and aspiration difficult at the site of the tumour. In 14 cases of squamous cell carcinoma lavage was also performed in the lung opposite to the tumour site. In patients of group 6 with pulmonary metastases lavage was carried out in territories where there was radiographic evidence of tumour.

\section{ALVEOLAR MACROPHAGE CHEMOTAXIS}

Cells were recovered by centrifugation and then washed and resuspended in phosphate buffered saline (PBS). Total and differential cell counts of an aliquot were performed on a cytocentrifuge preparation after Wright Giemsa staining. Cells were again centrifuged for 10 minutes at $500 \mathrm{~g}$, and finally resuspended at a concentration of $1.5 \times 10^{6}$ viable cells (trypan blue test) per ml of RPMI-1640 medium. Random migration and chemotaxis were measured in modified Boyden chambers with 8.0 $\mu \mathrm{m}$ pore millipore filters. "12 The 129 samples were tested in the presence of autologous zymosan activated serum $(1 \mathrm{mg}$ zymosan $/ \mathrm{ml}$ serum, one hour at $37^{\circ} \mathrm{C}$, then heated at $56^{\circ} \mathrm{C}$ for 30 minutes and diluted 1:20 in sterile PBS) as chemoattractant. Concomitantly, alveolar macrophages from six lung cancers were tested also against human $\mathrm{AB}$ zymosan activated serum, and samples from five lung cancers in the presence of $3 \times 10^{-8} \mathrm{mmol} / \mathrm{l}$ of formylmethonine-leucyl-phenylalanine (F-Met-Leu-Phe) per $\mathrm{ml}$. The number of macrophages migrating across the entire thickness of the filter after 90 minutes of incubation was determined after staining with haematoxylin. All the responding cells were in one optical plane on the lower surface of the filter, and the total number could be readily determined. The results are expressed as the mean cell count (with standard deviation in parentheses) in 10 microscopic fields $(\times 100)$ in duplicate assays. In 14 patients with sarcoidosis purified, lymphocyte free alveolar macrophages were obtained by allowing them to adhere to glass for 60 minutes, and the data were compared with those obtained with the total, unfractionated cell population.

STATISTICAL ANALYSIS

Differences between groups were compared by variance analysis $(F$ test). Means were compared with Student's $t$ test.

\section{Results}

The volume of recovered fluid, total cell yield, percentage of each cell type, and viability were not significantly different between the various groups (including healthy smokers and non-smokers) with the exception of patients with sarcoidosis, where the percentage of lymphocytes was higher than in the other groups (table 1).

The results shown in table 2 indicate that random migration performed as a control test in the presence of autologous serum diluted 1:20 in PBS was no different for patients with bronchial carcinoma or metastases from the result observed for healthy controls. Alveolar macrophages from patients with longstanding or recent bronchopulmonary infections, however, showed random migration significantly ( $p<0.02)$ above that of healthy controls.

Neither sex nor smoking habits showed any influence on alveolar macrophage chemotaxis in the healthy volunteer group. Chemotaxis was affected 
Table 1 General characteristics of recovered lavage fuids (means with standard deviations in parentheses)

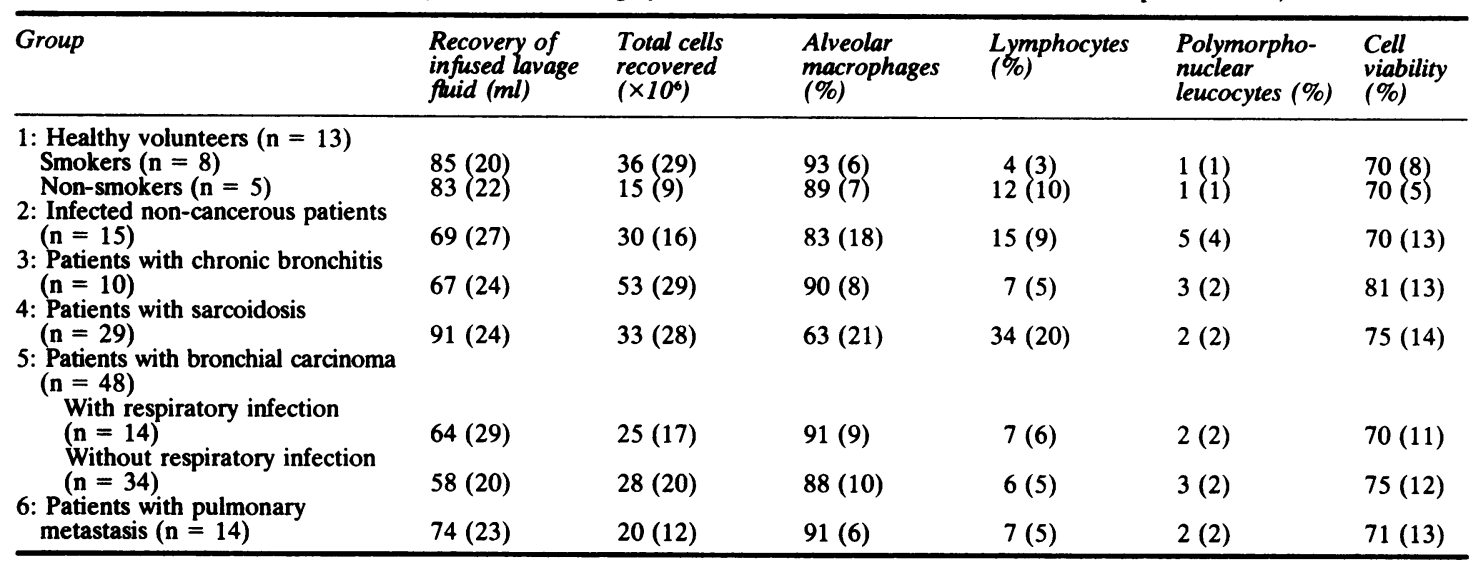

by clinical state (table 2 ); it was significantly less $(p<0.01)$ in patients with bronchogenic carcinoma than in controls, and there were no differences in values between cells recovered at the tumour site (mean 4.9 (SD 2.7)) and those from the homolateral lung in a territory close to the tumour $(5 \cdot 4(3.6))$. The dysfunction of alveolar macrophages from patients with primary bronchial carcinoma was independent of the histological type-squamous cell carcinoma yielded $4.5(1.7)$ cells per high powered field showing chemotactic migration, small cell cancers $5 \cdot 1(2 \cdot 1)$, adenocarcinomas $6 \cdot 1(2 \cdot 5)$, and undifferentiated tumours $7 \cdot 4(4 \cdot 8)$. The defect in alveolar macrophage activity was similar in patients with limited disease $(5.0(2.2))$ and patients with distant metastasis $(6 \cdot 3(4 \cdot 4))$. Interestingly, a con-

Table 2 Alveolar macrophage chemotaxis with autologous zymosan activated serum, expressed in terms of migrated alveolar macrophages (AM) per microscopic field (means with standard deviations in parentheses)

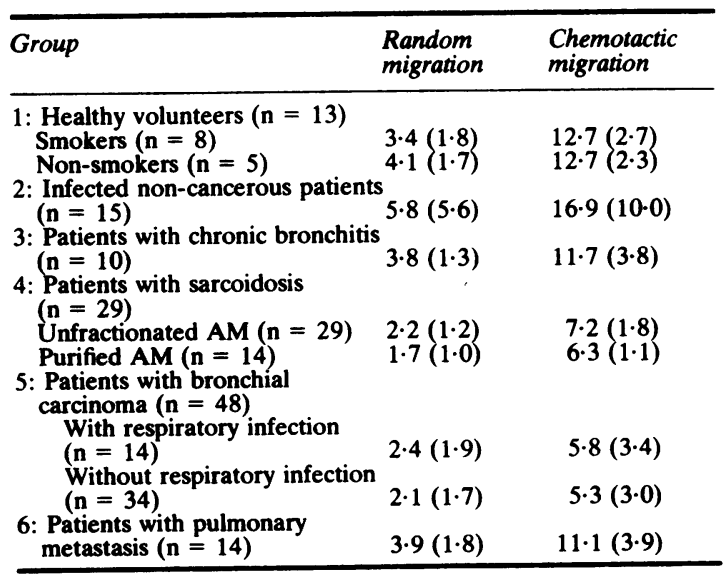

comitant respiratory infection or the finding of inflammatory bronchial mucosa did not affect low chemotactic migration values associated with a primary malignant lung tumour.

The use of various chemoattractants (zymosan treated autologous serum, zymosan treated $A B$ serum or F-Met-Leu-Phe) induced similar responses in the alveolar macrophages from patients with bronchogenic cancer (table 3 ). In contrast (table 2 ), the chemotaxis values of alveolar macrophages from patients with lung metastases of tumours from other sources (group 6) were similar to those of healthy controls (group 1). Defective alveolar macrophage chemotaxis was not, however, specific for primary bronchial carcinoma; the results for patients with endothoracic sarcoidosis (group 4) were similar to those for patients with bronchial carcinoma, showing chemotaxis significantly below $(p<0.01)$ that found in healthy controls. Table 2 also shows that depletion of lymphocytes before the test did not affect the data for sarcoidosis.

Chemotaxis was significantly more inhibited in alveolar macrophages from the tumour territory $(p<0.01)$ than in macrophages from the contra-

Table 3 Bronchial carcinoma: alveolar macrophage chemotaxis in the presence of either autologous zymosan activated serum, zymosan activated $A B$ serum, or F-Met-Leu-Phe (means with standard deviations in parentheses)

\begin{tabular}{lll}
\hline Chemotactic migration & & \\
\hline $\begin{array}{c}\text { Bronchogenic cancer } \\
\text { patients }(\mathrm{n}=6)\end{array}$ & $\begin{array}{c}\text { Autologous zymosan } \\
\text { activated serum } \\
\text { zymosan activated }\end{array}$ & $6.4(1 \cdot 3)$ \\
$\begin{array}{c}\text { AB serum } \\
\text { Bronchogenic cancer } \\
\text { patients }(\mathrm{n}=5)\end{array}$ & $\begin{array}{c}\text { Autologous zymosan } \\
\text { activated serum }\end{array}$ & $7 \cdot 1(2 \cdot 8)$ \\
F-Met-Leu-Phe & $\begin{array}{c}4.9 \\
5.8(1.9)\end{array}$ \\
\hline
\end{tabular}




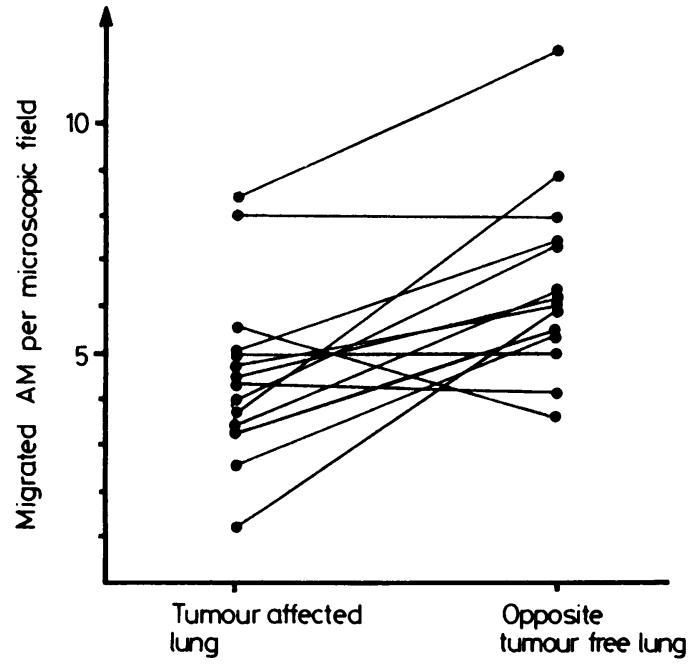

Bronchial carcinoma: alveolar macrophage (AM) chemotaxis with zymosan activated serum in the lung with the tumour and in the contralateral, tumour free lung.

lateral lung, although chemotaxis in the tumour free lung was still significantly below $(p<0.01)$ that of alveolar macrophages from healthy controls.

In contrast to the depressed alveolar macrophage chemotaxis associated with primary lung cancer or endothoracic sarcoidosis, chemotactic values were significantly greater in patients with a recent bronchopulmonary infection $(p<0.02)$ than in controls. Patients with chronic bronchitis without evidence of infection did not show any alteration in alveolar macrophage chemotaxis.

\section{Discussion}

When alveolar macrophages from healthy donors were tested for response to chemoattractants, only 10-15\% migrated. This contrasts sharply with results from mouse macrophage cell lines, where nearly $100 \%$ migration has been demonstrated. ${ }^{13}$ The lack of migration was not due to loss of cell viability as recovered non-migrating cells excluded trypan blue. Neither was it due to the chemoattractant, as the results were comparable in assays with zymosan activated autologous serum, zymosan activated human AB serum, and F-Met-Leu-Phe. The non-migrating population could not be explained by cell crowding or competition for pores on the filter. The finding suggests that alveolar macrophages could be made up of various subsets, although the migrating subset appeared to be independent of the chemotaxis agent, in contrast to blood monocyte subpopulations. ${ }^{14}$ is In the present series using alveolar macrophages from 32 women and 97 men, of whom 82 were smokers and 47 nonsmokers, no difference in responses were found that could be attributable to smoking habits or sex associated influences.

The data presented here indicate that alveolar macrophage chemotaxis was reduced in the presence of primary lung cancer and of sarcoidosis, in which cases inhibited alveolar macrophage responses were found to be independent of the presence or absence of lymphocytes in the sample studied in the chemotaxis chamber. This confirms a previous report. ${ }^{16}$ In contrast, normal values for alveolar macrophage chemotaxis were found in cells from patients with lung metastases, previously reported by Sone and Fidler. ${ }^{17}$ The finding might be related to the anatomical position of metastases.

Alveolar macrophages are situated at the airtissue interface, strategically located for initial contact with particulates and providing non-specific defence of the lung. Alveolar macrophages behave differently from blood monocytes and peritoneal macrophages in most activities, ${ }^{18}$ and may possess an autocontrol mechanism for local recruitment. ${ }^{19}$ Possibly metastases, made up of cells that have escaped capture by monocytes or macrophages in blood stream and lymph node and remaining separated from alveolar macrophages by a normal tissue barrier, are unable to deliver suppressive factors to the bronchial or alveolar lumen, whereas in a primary lung cancer developing on the bronchial epithelium there is cell to cell contact and free excretion of suppressive factors. Nevertheless, a study on a larger group of patients would be of interest, to confirm that alveolar macrophages from patients with lung metastases show a normal chemotactic response.

Alveolar macrophage chemotaxis was increased after a recent bronchopulmonary infection. This suggests an augmented mobility of alveolar macrophages induced by bacterial antigens. Augmented mobility was not seen in patients with bronchial carcinoma who had had recent bronchopulmonary infection. Mobility was presumably impaired by the concomitant presence of suppressor factors. Inhibitory factors released by tumour cells from sources other than the lung and impairing macrophage chemotaxis have been described ${ }^{2021}$ and a low molecular weight inhibitory factor has been isolated from growing neoplasia. ${ }^{22}$ A low molecular weight suppressor factor with prostaglandin like activity has, however, also been isolated from alveolar macrophages. $^{2324}$ Nearby lymphocytes might also be affected by reduced alveolar macrophage chemotaxis. It seems probable that suppressive factors are locally produced as chemotaxis was more inhibited in samples from the tumour territory than 
in samples from other lung areas.

Chemotactic responsiveness favours the accumulation of macrophages near the site of infection. It seems possible that the high incidence of bronchopulmonary infection in patients with lung cancer could be related to impaired alveolar macrophage chemotaxis. Accumulation of macrophages near the tumour is generally thought also to reflect participation in the defence against neoplasia, as impaired monocyte chemotaxis is found in about $60 \%$ of patients with cancers other than bronchogenic ones, and surgical removal is followed by restoration of chemotaxis to normal. ${ }^{59}$ It is tempting to speculate that an intrinsic functional defect of alveolar macrophages could favour the development and spread of primary lung cancer. The assay of alveolar macrophage chemotaxis could turn out to be a valuable diagnostic aid, especially when a peripheral tumour is not readily accessible to fibreoptic examination and biopsy, and perhaps particularly if it were associated with determination of the concentration of carcinoembryonic antigen in the lavage supernatant. ${ }^{25}$

This work was supported by contrat INSERM CRL 822010 .

\section{References}

' Hocking WG, Golde DW. The pulmonary alveolar macrophage. N Engl J Med 1979;301:580-7, 639-45.

${ }^{2}$ Kaltreider HB. Alveolar macrophages. Enhancers or suppressors of pulmonary immune reactivity. Chest 1982;82:261-2.

${ }^{3}$ Nathan CF, Murray HW, Cohn ZA. The macrophage as an effector cell. $N$ Engl J Med 1980;303:622-6.

${ }^{4}$ Hibbs JB, Chapman HA, Weinberg JB. The macrophage as an antineoplastic surveillance cell: biological perspectives. J Reticuloendothel Soc 1978;24:549-70.

${ }^{5}$ Boetcher DA, Leonard EJ. Abnormal monocyte chemotactic response in cancer patients. J Natl Cancer Inst 1974;52:1091-9.

${ }^{6}$ Normann SJ, Sorkin E. Inhibition of macrophage chemotaxis by neoplastic and other rapidly proliferating cells in vitro. Cancer Res 1977;37:705-11.

${ }^{7}$ Snyderman R, Pike MC, Altman LC. Abnormalities of leukocyte chemotaxis in human disease. Ann NY Acad Sci 1975;256:386.

${ }^{8}$ Snyderman R, Pike MC, Blaylock BL, Weinstein P. Effects of neoplasms on inflammation: depression of macrophage accumulation after tumour implantation. J Immunol 1976; 116:585-9.

${ }^{9}$ Snyderman R, Pike MC. Macrophage migratory dysfunction in cancer. Am J Pathol 1977;88:727-40.

${ }^{10}$ Reynolds HY, Fulmer JD, Kazmierowski JA, Roberts WC, Frank MM, Crystal RG. Analysis of cellular and protein content of bronchoalveolar lavage fluid from patients with idiopathic pulmonary fibrosis and chronic hypersensitivity pneumonitis. $J$ Clin Invest 1977;59:165-75.

"Snyderman R, Pike MC. Methodology for monocyte and macrophage chemotaxis In: Galin JI, Quie PG, eds. Leukocyte chemotaxis. New York: Raven Press, 1978:73-8.

12 Zigmond SH, Hirsch JG. Leukocyte locomotion and chemotaxis. J Exp Med 1973;137:387-410.

${ }^{13}$ Aksamit RR, Falk W, Leonard EJ. Chemotaxis by mouse macrophage cell lines. J Immunol 1981;126:2194-9.

${ }^{14}$ Ciancolo GJ, Snyderman R. Monocyte responsiveness to chemotactic stimuli is a property of a subpopulation of cells that can respond to multiple chemoattractants. $J$ Clin Invest 1981;67:60-88.

${ }^{15}$ Falk W, Leonard EJ. Human monocyte chemotaxis: migrating cells are a subpopulation with multiple chemotaxin specificities on each cell. Infect Immunol 1980;29:953-9.

${ }^{16}$ Lemarie E, Lavandier M, Legrand MF, Anthonioz P, Renoux M, Renoux G. Alveolar macrophage migration in pulmonary sarcoidosis. In: Chretien J, Marsac J, Saltiel JC, eds. Sarcoidosis and other granulomatous disorders. Paris: Pergamon Press, 1983:598-9.

${ }^{17}$ Sone S, Fidler J. Activation of rat alveolar macrophages to the tumoricidal state in the presence of progressively growing pulmonary metastases. Cancer Res 1981;4:2401-6.

${ }^{18}$ Shellito J, Caldwell JL, Kaltreider HB. Immune function of murine alveolar macrophages: binding of lymphocytes and support of lymphocyte proliferation. Exp Lung Res 1983;4:93-107.

${ }^{19}$ Privalova LI, Katsnelson BA, Osipenko AB, Yushkov BN, Babushkina LG. Response of a phagocyte cell system to products of macrophage breakdown as a probable mechanism of alveolar phagocytosis adaptation to deposition of particles of different cytotoxicity. Environ Health Perspect 1980;35:205-18.

${ }^{20}$ Pasternack GR, Snyderman R, Pike MC, Johnson RJ, Shin HS. Resistance of neoplasms to immunological destruction: role of a macrophage chemotactic inhibitor. J Exp Med 1978;148:93-102.

${ }^{21}$ Pike MC, Snyderman R. Depression of macrophage function by a factor produced by neoplasms: a mechanism for abrogation of immune surveillance. $J$ Immunol 1976;117:1243-9.

${ }^{22}$ Snynderman R, Pike MC. An inhibitor of macrophage chemotaxis produced by neoplasms. Science 976; 192:370-2.

${ }^{23}$ Holt PG. Alveolar macrophages. III: Studies on the mechanism of inhibition of $\mathrm{T}$-cell proliferation. Immunology 1979;34:437-45.

${ }^{24}$ McCombs CC, Michalski JP, Westerfield BT, Light RW. Human alveolar macrophages suppress the proliferative response of peripheral blood lymphocytes. Chest 1982;82:266-71.

${ }^{25}$ Lemarie E, Lavandier M, Renoux M, Renoux G Carcinoembryonic antigen is increased in bronchoalveolar lavage fluid of lung cancer. In: Pontifex J, ed. Lung cancer: etiology, epidemiology, prevention, early diagnosis, treatment. Amsterdam: Excerpta Medica, 1981:244-51. 